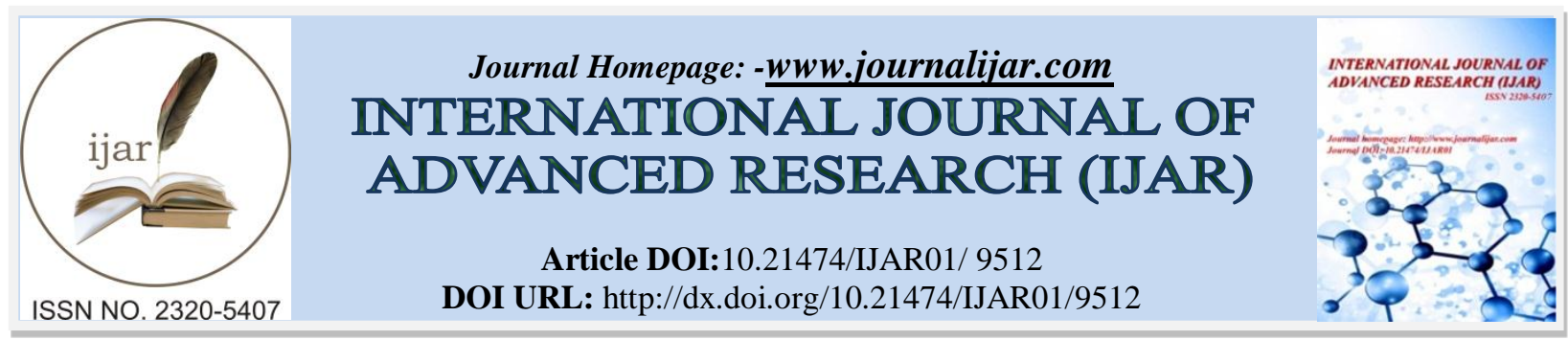

ORIGINAL ARTICLE

\title{
ADJUVANT TREATMENT WITH REPETITIVE TRANS CRANIAL MAGNETIC STIMULATION IN FRESHLY DIAGNOSED PATIENTS OF MAJOR DEPRESSION IN INDIAN POPULATION - AN OUTCOME STUDY.
}

\author{
Dr. Anindya Kumar Gupta ${ }^{1}$ and Dr. Amit Kumar ${ }^{2}$. \\ 1. Associate Professor (Psychiatry), Command Hospital (AF) Bangalore, India. \\ 2. Associate Professor (Psychiatry), Command Hospital (AF) Bangalore, India.
}

\section{Manuscript Info}

Manuscript History

Received: 06 June 2019

Final Accepted: 08 July 2019

Published: August 2019

\section{Abstract}

Copy Right, IJAR, 2019,. All rights reserved.

\section{Introduction:-}

Transcranial magnetic stimulation (TMS) is a neuro-stimulation and neuromodulation technique, based on the principle of electromagnetic induction of an electric field in the brain (1-2). This has behavioral consequences and therapeutic potential (3). Repetitive transcranial magnetic stimulation (rTMS) has been found to be a promising noninvasive treatment for a variety of neuropsychiatric conditions and the number of applications continues to increase with a large number of ongoing clinical trials in a variety of diseases(4-10). In October 2008, a specific rTMS device was approved by the Food and Drug Administration in the United States for the treatment of patients with medication-refractory unipolar depression who have failed one good (but not more than one) pharmacological trial (11). Depressive disorders in various forms have been in the forefront of the disease burden in the world. Every year, a significant number of lives are lost and/or disabled due to depressive disorders (12). To best of our knowledge, little has been done so far to understand use of rTMS treatment as an adjunct in freshly diagnosed major depression (13-16) and specifically in indian population (17-19). Hence the current study is an open label study to explore the same and also to evaluate its safety and tolerability in indian context.

\section{Methods:-}

In a prospective, open label study design; forty freshly diagnosed cases of major depressive were included. After informed consent, half of them were given adjuvant rTMS along with standard treatment \& rest were treated only with the standard treatment but without rTMS after suitable randomization. The DSM-5 Diagnostic Criteria were followed to establish diagnosis ratified by both Principal worker and Co-Worker (both psychiatrists) (20). Drug Treatment was as per standard treatment guidelines issued by Maudsley Guidelines UK (21) and rTMS were given as per recommended protocols published by NICE guidelines 2015 (UK) (22) as Indian protocols do not exist at present. Outcome of treatment was decided based on the psychometric test (MADRAS) (23). Blinding was not practiced as the effects or side effects of rTMS were not possible to be concealed. Previously diagnosed patients of psychiatric and medical illnesses were excluded. Individuals with a history of seizures or serious medical conditions did not participate in the study. Pediatric population was excluded and patients who have been treated by rTMS earlier were excluded. Relevant statistical methods were used for analysis.

Corresponding Author:-Dr. Amit Kumar

Address:-Associate Professor, Dept of Psychiatry, Command Hospital (AF) Bangalore, Agram Post, Bengaluru (Karnataka, India). 


\section{Results:-}

\section{Patient Groups}

A total of 40 patients, 12 males and 28 females, participated (age range 18-50years) (Distribution as per chart 1 and 2). All the patients had a diagnosis of fresh major depression. Ten patients met the criteria for comorbid social phobia, six generalized anxiety disorder, and four obsessive compulsive disorder (Distribution as per chart 3, 4 and 5). A total of 18 of 20 patients in the test group and 16 of the 20 in the control group cont inued for the full 4 weeks of treatment. Other patients discontinued after 2-3 weeks. The reasons for withdrawal included unrelated medical illness (one in standard group), family commitments (one each in standard and test group) and lack of response (two in control and one in test group).

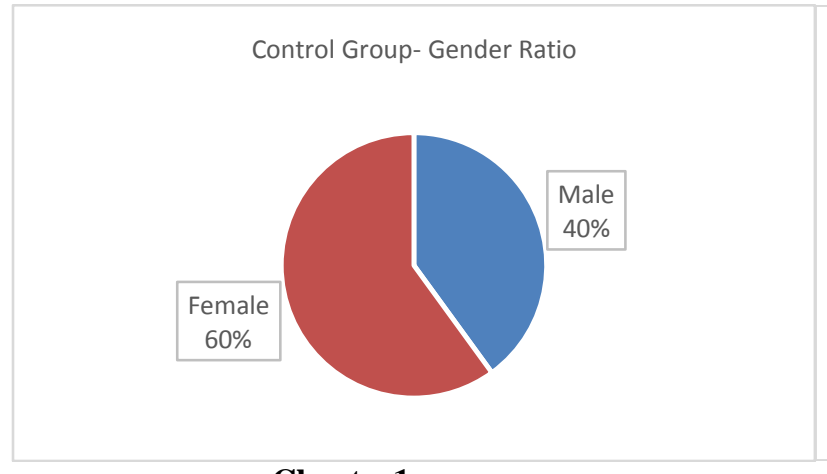

Chart - 1

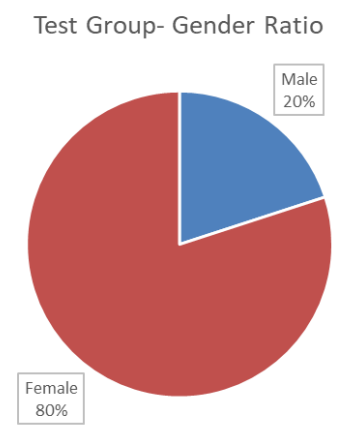

Chart - 2

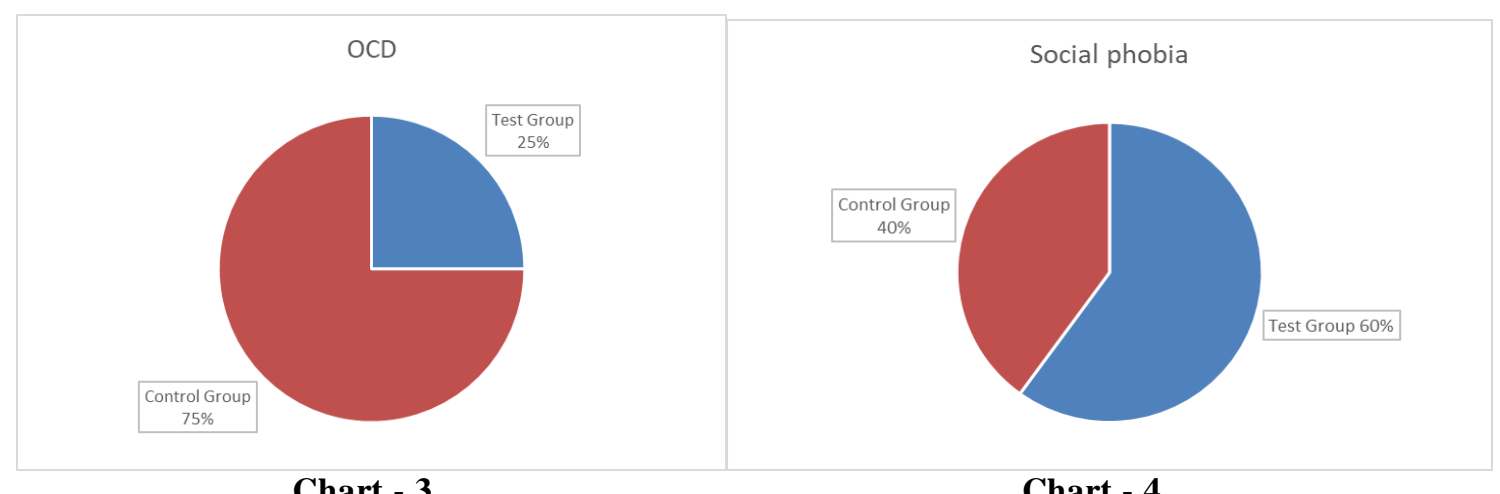

Chart - 3

\section{Chart - 4}

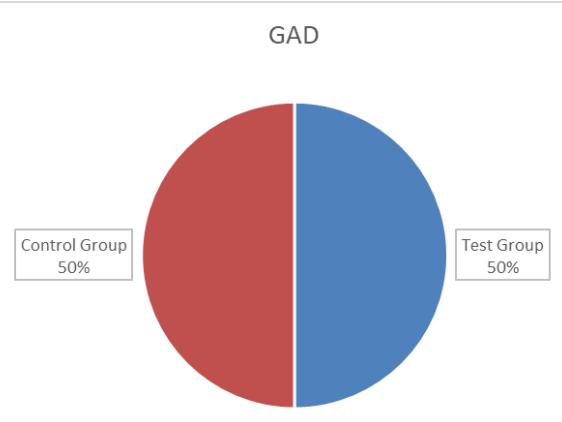

Chart - 5 


\section{Outcome}

The MADRS score was analyzed to at 3 different stages. At the beginning, the results indicated that there was no statistical difference in the means of test and control group. $(p>0.05)$. So the experiment was started at the same level for both the groups. After 2 weeks and 4 weeks, as per the planned testing, the MADRS sores were again tested. At the end of 4 weeks, mean difference was found to be statistically significant indicating that test group has improved much faster than the control group $(\mathrm{p}<0.05)$.

t-Test:

\begin{tabular}{|l|r|r|}
\hline & \multicolumn{1}{|c|}{ Control } & \multicolumn{1}{|c|}{ Test } \\
\hline Mean & 29.25 & 29.2 \\
\hline Variance & 12.618 & 13.747 \\
\hline Observations & 20 & 20 \\
\hline Hypothesized Mean Difference & 38 & \\
\hline $\mathrm{df}$ & 0.044 & \\
\hline $\mathrm{t}$ Stat & 0.965 & \\
\hline $\mathrm{p}(\mathrm{T}<\mathrm{t})$ two-tail & 2.024 & \\
\hline $\mathrm{t}$ Critical two-tail & & \\
\hline \hline \multicolumn{2}{|c|}{ Hypothesis testing for mean difference at baseline } & \\
\hline
\end{tabular}

\section{t-Test:}

\begin{tabular}{|c|c|c|}
\hline & Control & Test \\
\hline Mean & 23.35 & 21.3 \\
\hline Variance & 12.344 & 13.589 \\
\hline Observations & 20 & 20 \\
\hline Hypothesized Mean Difference & 0 & \\
\hline df & 38 & \\
\hline t Stat & 1.800 & \\
\hline $\mathrm{p}(\mathrm{T}<=\mathrm{t})$ two-tail & 0.079 & \\
\hline t Critical two-tail & 2.024 & \\
\hline Hypothesis testing for mean difference at two weeks & & \\
\hline
\end{tabular}

t-Test:

\begin{tabular}{|c|c|c|}
\hline & Control & Test \\
\hline Mean & 14.6 & 10.65 \\
\hline Variance & 8.568 & 7.186 \\
\hline Observations & 20 & 20 \\
\hline Hypothesized Mean Difference & 0 & \\
\hline df & 38 & \\
\hline t Stat & 4.450 & \\
\hline $\mathrm{p}(\mathrm{T}<=\mathrm{t})$ two-tail & 7.266 & \\
\hline t Critical two-tail & 2.024 & \\
\hline Hypothesis testing for mean difference at four weeks & & \\
\hline
\end{tabular}




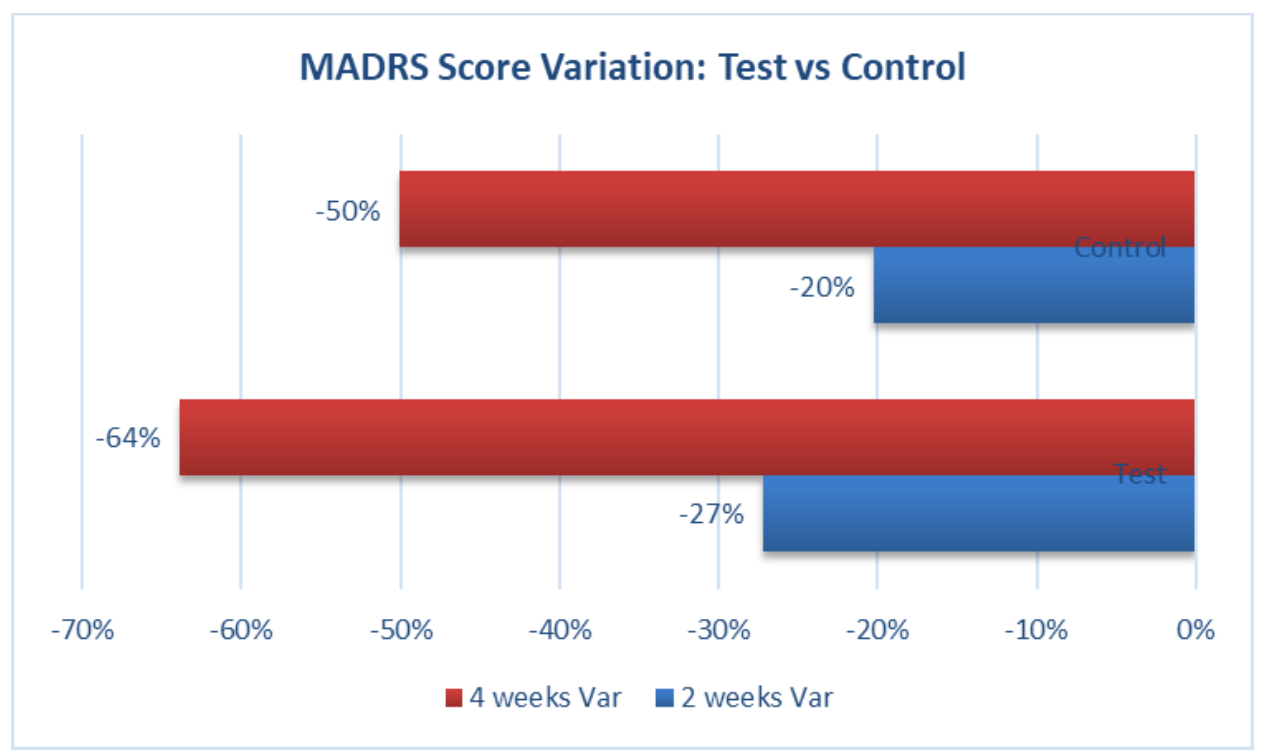

Fig 1:-Test versus control group: 2 weeks and 4 weeks

All the patients tolerated the treatment well without any report of adverse effects. Adequate safety measures were ensured to manage unexpected seizures while treatment was being delivered.

\section{Discussion:-}

We have analyzed our results based on evidence prior to the study period, which gives more value to the conclusions that are drawn below. This prospective open label study on forty freshly diagnosed patients of major depression explored outcome in indian population after treatment with standard protocols vs treatment with standard protocols along with adjuvant therapy with repititative transcranial magnetic stimulation for a period of six month.

The patients were divided into two matched groups of twenty patients each which were randomly assigned for treatment with or without rTMS after applying the exclusion and inclusion criteria described earlier. After the initial treatment, they were followed up periodically at two weeks and four weeks intervals and their outcomes were documented. Analysis of the data clearly showed that the test group with rTMS had a better outcome compared to the control group of standard treatment for major depression. There is growing and relatively consistent evidence that standard high-frequency left-sided rTMS treatment is more effective in the treatment of depression than sham stimulation (24). However, the degree of clinical response seen in most trials has been limited.

\section{Limitations of the study}

It is important to note that the main findings of this study are limited by a difference in dropout rates experienced by the two groups. Given the structure of the trial, it is likely that the greater dropout rate in the standard group was related to poorer overall response in these patients. This is reflected in the larger proportion of subjects in the non-response category for dropout in the standard group. There is certainly a strong indication that targeted treatment was associated with a reduced dropout because of the lack of efficacy supporting the notion that this intervention was more effective. It is also noted that this imbalance in dropout rates would actually bias towards a smaller, between-group difference at 4 weeks. Therefore, the difference in dropouts does affect our capacity to interpret the 4-week results in the analysis.

There are some other limitations of the clinical trial worthy of mention. Firstly, we have provided treatment for 4 weeks; studies are now testing rTMS methods over longer periods of time (25). It is possible that the standard group could 'catch up' in their response rates with a longer duration of treatment. Secondly, the patients in our study had a high rate of comorbid anxiety group of disorders. Although mood and anxiety disorders are frequently comorbid, it is possible that the response rates to rTMS in general may be different in a group of patients with a single disorder, perhaps of the melancholic depression type (24). However, this effect does require replication in larger samples. 
One of the major limitation in this study was the smaller sample size in this study. Many factors were not considered which may have influenced our findings like psycho social stressors etc.

\section{Future implications}

If the efficacy of rTMS in treating depression needs to be established on a par with other existing antidepressant modalities, the above-mentioned difficulties must be overcome, and if this can be done, this procedure is definitely a better option considering the following: its very good safety profile; it is well tolerated and convenient to use; it does not need anaesthesia of ECT; and it is easy to administer for the clinician. This study is one of the few of its kind in india and there is definitely a positive trend toward its use in depression and it is worthwhile conducting more trials using larger sample sizes and more rigorous research design in our setting.

\section{Conclusion:-}

This study attempted to find out the efficacy of rTMS as an adjunct in the treatment of freshly diagnosed major depression which was statistically significant. Other aim was to evaluate its safety and tolerability in indian population. The intervention is easily administered, non-invasive and free of any major side-effects, hence the acceptance rate among patients is high and drop-out rates are very few.

\section{Declaration of Interests:}

Nil

\section{References:-}

1. Barker A, Freeston I, Jalinous R, Jarrat J. Non invasive magnetic stimulation of the human motor cortex. Lancet $1985 ; 2: 1106-7$.

2. Barker AT. The history and basic principles of magnetic nerve stimulation. Electroencephalogr Clin Neurophysiol Suppl 1999; 51:3-21.

3. Chen R, Cros D, Curra A, Di Lazzaro V, Lefaucheur JP, Magistris MR, et al. The clinical diagnostic utility of transcranial magnetic stimulation.

4. Lefaucheur JP. Principles of therapeutic use of transcranial and epidural cortical stimulation. Clin Neurophysiol 2008; 119:2179-84.

5. Siebner HR, Rothwell J. Transcranial magnetic stimulation: new insights into representational cortical plasticity. Exp Brain Res 2003; 148:1-16.

6. Lefaucheur JP. Methods of therapeutic cortical stimulation. Neurophysiol Clin 2009; 39:1-14.

7. Heinz A, Beck A, Grusser M, Grace AA, Wrase J. Identifying the neural circuitry of alcohol craving and relapse vulnerability. Addict Biol 2009; 14:108-18.

8. Goldstein RZ, Volkow ND. Drug addiction and its underlying neuro-biological basis: neuroimaging evidence for the involvement of the frontal cortex. Am J Psychiatry 2002; 159:1642-52.

9. Wilson SJ, Sayette MA, Fiez JA. Prefrontal responses to drug cues: a neurocognitive analysis. Nat Neurosci 2004; 7:211-4.

10. Makani R, Pradhan B, Shah U, Parikh T. Role of repititative transcranial magnetic stimulation(rTMS) in treatment of addiction and related disorders: a systemic review. Curr drug Abuse Rev 2017; 10(1):31-43.

11. Slotema CW, Blom JD, Hoek HW, Sommer IE (July 2010). "Should we expand the toolbox of psychiatric treatment methods to include Repetitive Transcranial Magnetic Stimulation (rTMS)? A meta-analysis of the efficacy of rTMS in psychiatric disorders". The Journal of Clinical Psychiatry. 71 (7): 873-84.

12. 12.FerrariAJ, CharlsonFJ, NormanRE, etal.Burden of depressive disorders by country, sex, age, and year: findings from the global burden of disease study2010.PLoSMed.2013;10(11):e1001547.

13. 13.LevkovitzY, IsserlesM, PadbergF,etal.Efficacy and safety of deep trans cranial magnetic stimulation for major depression: aprospective multi center randomized controlled trial. World Psychiatry.2015;14(1):64-73.

14. BakkerN, ShahabS, GiacobbeP, etal. Rtms of the dorsomedial prefrontal cortex for major depression: safety, tolerability, effectiveness, and outcome predictors for $10 \mathrm{~Hz}$ versus intermittent theta-burststimulation.Brain Stimul.2015;8(2): 208-215.

15. LeuchterAF, CookIA, FeifelD, etal. Efficacy and safety of low-field synchronized transcranial magnetic stimulation(sTMS) for treatment of major depression.BrainStimul.2015;8(4):787-794.

16. FitzgeraldPB, HoyK, McQueenS, etal. Priming stimulation enhances the effectiveness of low-frequency right prefrontal cortex transcranial magnetic stimulation in major depression. JClin Psychopharmacol.2008;28(1):5258. 
17. Lingeswaran A. Repetitive transcranial magnetic stimulation in the treatment of depression: A randomized, double-blind, placebo-controlled trial. Indian J Psychol Med. 2011;33:35-44.

18. Nongpiur A, Sinha VK, Praharaj SK, Goyal N. Theta-patterned, frequency-modulated priming stimulation enhances low-frequency, right prefrontal cortex repetitive transcranial magnetic stimulation (rTMS) in depression: A randomized, sham-controlled study. J Neuropsychiatry Clin Neurosci. 2011;23:348-57.

19. Ray S, Nizamie SH, Akhtar S, Praharaj SK, Mishra BR, Zia-ul-Haq M. Efficacy of adjunctive high frequency repetitive transcranial magnetic stimulation of left prefrontal cortex in depression: A randomized sham controlled study. J Affect Disord. 2011;128:153.

20. 20. American Psychiatric Association. (2013). Diagnostic and statistical manual of mental disorders (5th ed.). Washington, DC:

21. The Maudsley Prescribing Guidelines in Psychiatry, 13th Edition. Wiley.com. 18 May 2018. Retrieved 21 May 2018.

22. Rossi S. Safety of transcranial magnetic stimulation: With a note on regulatory aspects. In: Miniussi C, Paulus W, Rossini PM, editors. Transcranial brain stimulation Boca Raton, FL: CRC Press, Taylor \& Francis Group; 2013. p. 415-26.

23. Montgomery SA, Asberg M (April 1979). "A new depression scale designed to be sensitive to change". British Journal of Psychiatry. 134 (4): 382.

24. Fitzgerald, P. B., Brown, T. L., Marston, N. A. U., Daskalakis, Z. J., de Castella, A., \& Kulkarni, J. (2003). Transcranial magnetic stimulation in the treatment of depression: A double-blind, placebo-controlled trial. Archives of General Psychiatry, 60(10), 1002.

25. Fitzgerald, P. B., Benitez, J., de Castella, A., Daskalakis, Z. J., Brown, T. L., \& Kulkarni, J. (2006). A randomized, controlled trial of sequential bilateral repetitive transcranial magnetic stimulation for treatmentresistant depression. The American Journal of Psychiatry, 163(1), 88-94. doi:10.1176/appi.ajp.163.1.88. 